\title{
ANGPTL4 overexpression is associated with progression and poor prognosis in breast cancer
}

\author{
JING ZHAO, JUNTIAN LIU, NAN WU, HAILIAN ZHANG, \\ SHICHAO ZHANG, LIJUAN LI and MENG WANG
}

Department of Breast Cancer, Cancer Institute and Hospital, Tianjin Medical University, Tianjin 360000, P.R. China

Received October 1, 2019; Accepted April 23, 2020

DOI: $10.3892 /$ ol.2020.11768

\begin{abstract}
The aim of the present study was to analyze the expression levels of angiopoietin-like 4 (ANGPTL4) in breast cancer to investigate the association between ANGPTL4 and breast cancer. Immunohistochemistry was performed on formalin-fixed paraffin-embedded tissues, including 205 invasive ductal carcinoma (IDC) of no special type, 40 normal breast, 40 atypical ductal hyperplasia (ADH) and 40 ductal carcinomas in situ (DCIS) tissues. The non-parametric Kruskal-Wallis test was used to evaluate the differential expression of ANGPTL4 and clinicopathological parameters in breast cancer. Kaplan-Meier analysis and Cox regression analysis were used to evaluate the association between the expression levels of ANGPTL4 and the prognosis of breast cancer. The results revealed that ANGPTL4 expression was higher in IDC $(63.4 \% ; 130 / 205)$ compared with in normal breast tissues $(17.5 \% ; 7 / 40), \mathrm{ADH}(30 \% ; 12 / 40)$ and DCIS (37.5\%; 15/40). The clinical significance of ANGPTL4 expression was analyzed in a total of 205 IDC tissues, and high expression levels of ANGPTL4 were positively associated with pathological stage $(\mathrm{P}<0.001)$, tumor size $(\mathrm{P}<0.001)$, histological grade $(\mathrm{P}<0.001)$, lymph node metastasis $(\mathrm{P}<0.001)$, distant metastasis $(\mathrm{P}<0.001)$ and local recurrence $(\mathrm{P}<0.001)$. Kaplan-Meier analysis revealed that patients with high ANGPTL4 expression had a shorter overall survival (OS; $\mathrm{P}<0.001$ ) and disease-free survival (DFS; $\mathrm{P}<0.001$ ) compared with patients with low ANGPTL4 expression. Multivariate Cox regression analysis revealed that ANGPTL4 was an independent prognostic factor for breast cancer $\mathrm{OS}(\mathrm{P}=0.034)$ and DFS $(\mathrm{P}=0.011)$. The results of the present study demonstrated that ANGPLT4 was associated with malignant progression and poor prognosis of breast cancer, suggesting that ANGPLT4 may be a novel therapeutic target for breast cancer.
\end{abstract}

Correspondence to: Dr Meng Wang, Department of Breast Cancer, Cancer Institute and Hospital, Tianjin Medical University, West Huanhu Road, Hexi, Tianjin 360000, P.R. China

E-mail: wangmeng312@126.com

Key words: angiogenesis, angiopoietin-like 4, breast cancer, prognosis

\section{Introduction}

Breast cancer is a complex and heterogeneous disease, and it is the most common type of cancer and the most frequent cause of cancer-associated mortality among women worldwide $(1,2)$. Although there has been a rapid development of therapeutic modalities that resulted in a $\sim 38 \%$ decreased mortality rate, patients with metastasis and local recurrence continue having a poor prognosis (3). The degree of malignancy, prognosis and treatment failure is associated with numerous factors, including the existence of stem cells, GTPases and angiogenesis (4-6). Angiopoietin-like 4 (ANGPTL4) is a protein belonging to the ANGPTL family that was discovered in $2000(7,8)$. ANGPTL4 is involved in regulating angiogenesis, fat metabolism, energy homeostasis, redox reactions, inflammation and endothelial cell integrity, which is associated with complex protein structures $(9,10)$. ANGPTL4 serves an important role in tumor-associated activities, such as tumor cell motility and invasiveness, cell migration, endothelial cell function, vascular leakage, neoangiogenesis and cell adhesion and motility, by interacting with matrix proteins in a variety of solid tumors (9). ANGPTL4 has been reported to prevent lung carcinoma and melanoma metastasis via the inhibition of vascular permeability and tumor cell motility and invasiveness (11). By contrast, Chen et al (12) reported that ANGPTL4 is involved in gastric cancer (GC) cell proliferation, apoptosis and metastasis in vitro, suggesting that it may act as a novel potential therapeutic target for patients with GC. High ANGPTL4 expression is associated with poor prognosis in oral cancer (13). Conversely, ANGPTL4 expression is lower in hepatocellular carcinoma tissues than in non-tumor tissues (14). For other tumors, such as colorectal cancer and renal cell cancer, studies have reported that ANGPTL4 promotes venous invasion and distant metastasis $(15,16)$. Although ANGPTL4 appears to serve a controversial role in a number of tumors, there is currently no effective targeted therapy against ANGPTL4.

ANGPTL4 induces obesity-associated metabolic disorders through various insulin signaling pathway-associated genes (17). One of the most important signaling pathways is the PI3K/AKT signaling pathway, which can improve systemic glucose tolerance and simultaneously induce hypertriglyceridemia and liver steatosis (18). The PI3K/AKT signaling pathway has been intensively investigated in cancer due to its important role in cell survival and anti-apoptotic mechanisms. 
A previous study has demonstrated that the AKT signaling pathway is frequently altered in numerous types of cancer and, in some cases, is associated with tumor aggressiveness (19). A number of cancer types, including breast cancer, are associated with AKT upregulation. The importance of AKT in breast cancer makes it an attractive target for anticancer therapy, and multiple drug discovery programs are focused on finding selective and potent AKT inhibitors $(18,20)$. Several compounds, including GSK2141795, AZD5363 and Perifosine, have been demonstrated to inhibit AKT in in vitro and in vivo models, and some are currently under clinical evaluation $(18,20)$.

Nevertheless, the role of ANGPTL4 in breast cancer remains unclear. In the present study, the association between ANGPTL4 expression and breast cancer was investigated. ANGPTL4 expression was examined in normal, atypical ductal hyperplasia (ADH), ductal carcinoma in situ (DCIS) and invasive ductal carcinoma (IDC) tissues, and its prognostic value was analyzed in patients with breast cancer, revealing an association between ANGPTL4 expression and breast cancer.

\section{Materials and methods}

Patient samples. All patients in the present study were female. IDC of no special type tissue specimens were consecutively collected from surgical resections of 205 female patients who underwent mammectomy at the Cancer Hospital of Tianjin Medical University (Tianjin, China) between January 1, 2003 and December 31, 2003. A total of 40 normal breast tissues were selected randomly and taken from the distal end of the IDC surgical specimens (mean age, 50 years; range, 28-70 years), 40 ADH specimens were taken from the adjacent tissues $(\geq 1 \mathrm{~cm}$ away) of DCIS surgical specimens (confirmed as ADH by pathological examination; mean age, 45 years; range, $28-65$ years) and 40 DCIS specimens (mean age, 48 years; range, 28-67 years) were selected randomly from the surgical specimens of 896 patients diagnosed with DCIS at the Cancer Hospital of Tianjin Medical University during the aforementioned period, and were used as controls. The present study followed a retrospective approach. The clinicopathological characteristics, including age, histological grade, lymph node metastasis, distant metastasis, pathological stage according to the American Joint Committee on Cancer staging (21) and follow-up, were evaluated by reviewing medical charts and pathological records from the Department of Breast Cancer Pathology and Research Laboratory (Cancer Hospital of Tianjin Medical University). The patients had a median age of 55 years (range, 25-81 years) and a median follow-up period of 54 months (range, 30-71 months). Overall survival (OS) was defined as the time from the date of surgery to the date of mortality. Disease-free survival (DFS) was defined as the time from the date of surgery to the date of recurrence. Cases lost to follow-up or death by causes other than breast cancer were censored in the survival analysis. Patients with incomplete pathological and clinical information, or recurrent patients were excluded. None of the patients received preoperative radiation or chemotherapy. The present study was approved by the Ethics Committee of the Cancer Institute \& Hospital of Tianjin Medical University (Tianjin, China; approval no. EK2012053). 

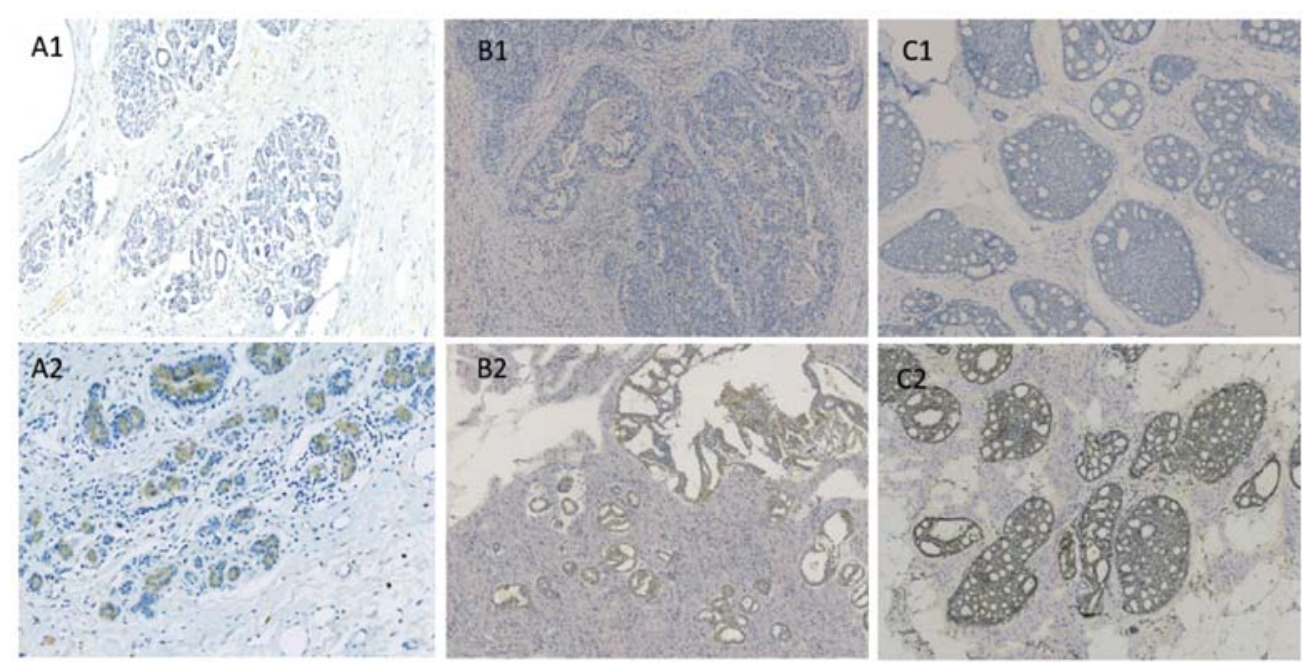

Figure 1. The IHC staining of angiopoietin-like 4 expression in normal breast, atypical ductal hyperplasia and ductal carcinoma in situ tissues. Angiopoietin-like 4 in normal breast tissues: (A1) Negative, (A2) positive; in atypical ductal hyperplasia tissues: (B1) Negative, (B2) positive; and in ductal carcinoma in situ tissues: (C1) Negative, (C2) positive. Magnification, x200.

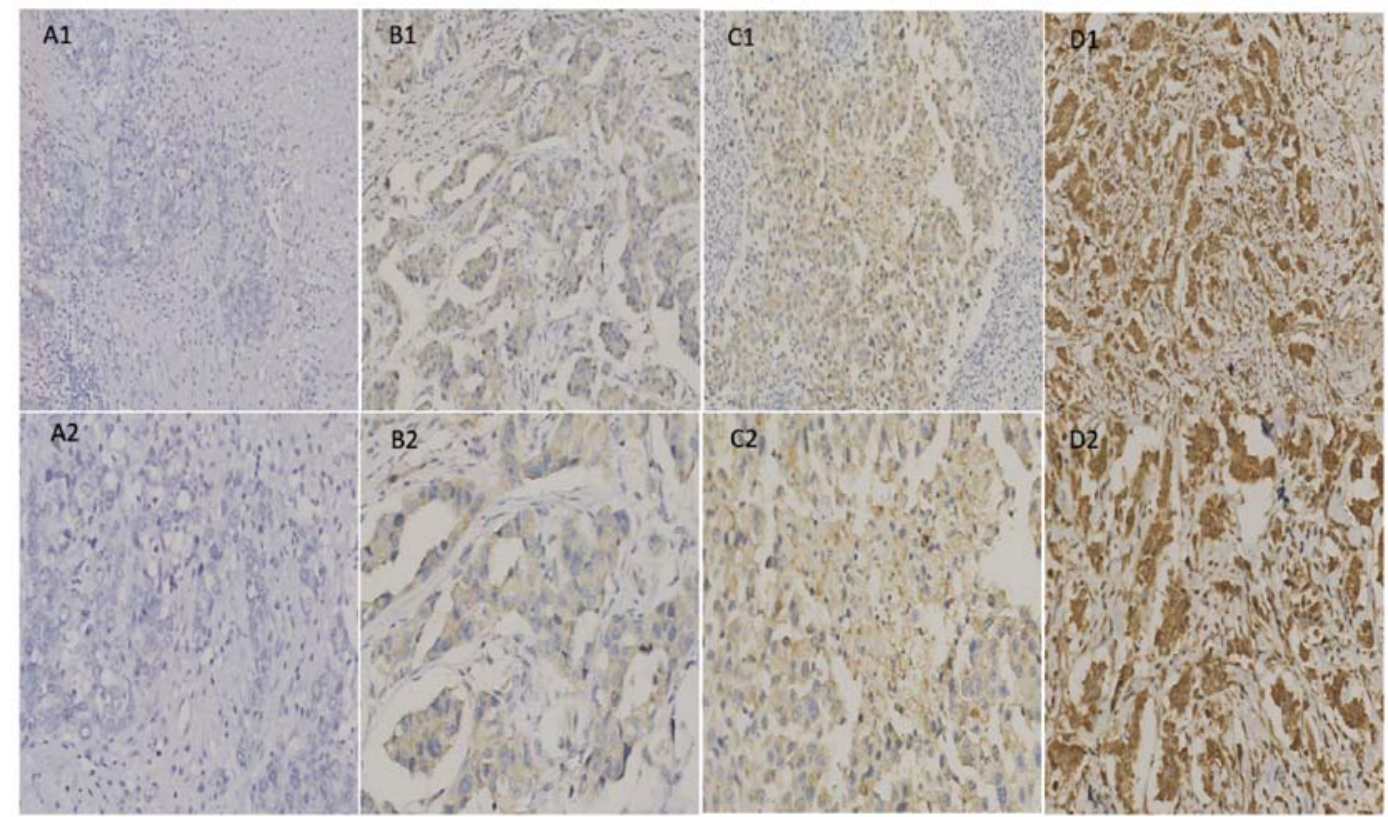

Figure 2. Angiopoietin-like 4 expression in invasive ductal carcinoma. (A1) Negative (magnification, x200); (A2) negative (magnification, x400); (B1) weakly positive (magnification, x200); (B2) weakly positive (magnification, x400); (C1) moderately positive (magnification, x200); (C2) moderately positive (magnification, $\mathrm{x} 400$ ); (D1) strongly positive (magnification, $\mathrm{x} 200$ ); (D2) strongly positive (magnification, $\mathrm{x} 400$ ).

40 normal breast, $40 \mathrm{ADH}$ and 40 DCIS tissues. The tissue samples were fixed in $10 \%$ formalin for $24 \mathrm{~h}$ at room temperature and embedded in paraffin. Tissue sections (4- $\mu \mathrm{m}$-thick) were deparaffinized in xylene and rehydrated in a descending ethanol series $(100,95,80$ and $70 \%)$ at room temperature. Following de-waxing and hydration, $4-\mu \mathrm{m}$ sections were treated with EDTA buffer solution (pH 9.0) using pressure cooking for $2 \mathrm{~min}$. Endogenous peroxidase activity was inactivated by incubation with $3 \% \mathrm{H}_{2} \mathrm{O}_{2}$ for 10 min at room temperature. Non-specific binding sites were blocked by incubation with $10 \%$ normal goat serum (cat. no. ab7481; Abcam) at room temperature for $20 \mathrm{~min}$. Tissue sections were then incubated with a primary ANGPTL4 polyclonal antibody (dilution, 1:100; cat. no. A01147; Boster Biological Technology) at $37^{\circ} \mathrm{C}$ for $2 \mathrm{~h}$. All sections were subsequently incubated using the Dako REAL ${ }^{\mathrm{TM}}$ EnVision $^{\mathrm{TM}}$ Detection System (cat. no. K5007; Dako; Agilent Technologies, Inc.) containing biotinylated anti-rabbit secondary antibodies (dilution, 1:100) for $20 \mathrm{~min}$ at $37^{\circ} \mathrm{C}$, and peroxidase-labelled streptavidin for $20 \mathrm{~min}$ at room temperature (dilution, 1:100). For the negative control, the primary antibody was replaced by PBS. All sections were stained with 3,3'-diaminobenzidine tetrahydrochloride at room temperature for $15 \mathrm{sec}$.

Samples were considered positive for ANGPTL4 if cytoplasmic immunoreactivity was observed in the tumor cells. Protein expression was evaluated using a semi-quantitative system after a series was examined on a double-headed light microscope with magnifications of $\times 5, \times 10, \times 20$ and $\times 40$. The 
Table II. Association between ANGPTL4 expression and clinical parameters in 205 patients with invasive ductal carcinoma.

ANGPTL4 expression, $\mathrm{n}$

\begin{tabular}{|c|c|c|c|c|c|}
\hline \multirow[b]{2}{*}{ Variable } & & \multirow[b]{2}{*}{ P-value } \\
\hline & Negative & Weakly positive & Moderately positive & Strongly positive & \\
\hline Age, years & & & & & 0.371 \\
\hline$<55$ & 38 & 28 & 30 & 12 & \\
\hline$\geq 55$ & 37 & 25 & 22 & 13 & \\
\hline Tumor size, $\mathrm{cm}$ & & & & & $<0.001$ \\
\hline$\leq 2.0$ & 46 & 10 & 15 & 7 & \\
\hline $2.1-5.0$ & 29 & 41 & 30 & 8 & \\
\hline$>5.0$ & 0 & 2 & 7 & 10 & \\
\hline pTNM & & & & & $<0.001$ \\
\hline I & 30 & 6 & 4 & 0 & \\
\hline II & 29 & 38 & 28 & 7 & \\
\hline III & 16 & 7 & 14 & 12 & \\
\hline IV & 0 & 2 & 6 & 6 & \\
\hline Grade & & & & & $<0.001$ \\
\hline I & 25 & 8 & 4 & 0 & \\
\hline II & 34 & 31 & 31 & 15 & \\
\hline III & 16 & 14 & 17 & 10 & \\
\hline ER & & & & & 0.955 \\
\hline- & 38 & 29 & 28 & 12 & \\
\hline+ & 37 & 24 & 24 & 13 & \\
\hline PR & & & & & 0.793 \\
\hline- & 41 & 30 & 22 & 18 & \\
\hline+ & 34 & 23 & 30 & 7 & \\
\hline HER 2 & & & & & 0.211 \\
\hline- & 35 & 32 & 22 & 9 & \\
\hline $1+$ & 16 & 5 & 18 & 8 & \\
\hline $2+$ & 14 & 4 & 6 & 4 & \\
\hline $3+$ & 10 & 12 & 6 & 4 & \\
\hline Lymph node metastasis & & & & & $<0.001$ \\
\hline- & 51 & 29 & 25 & 6 & \\
\hline+ & 24 & 24 & 27 & 19 & \\
\hline Distant metastasis & & & & & $<0.001$ \\
\hline- & 68 & 46 & 37 & 15 & \\
\hline+ & 7 & 7 & 15 & 10 & \\
\hline Local recurrence & & & & & \\
\hline- & 75 & 51 & 48 & 19 & $<0.001$ \\
\hline+ & 0 & 2 & 4 & 6 & \\
\hline
\end{tabular}

ER, estrogen receptor; PR, progesterone receptor; HER2, human epidermal growth factor receptor 2; ANGPTL4, angiopoietin-like 4.

staining was scored independently by two pathologists blinded to patients' outcomes. The immunoreactive intensity of each sample was scored by two pathologists as follows: 0 , negative; 1, low; 2, medium; and 3, high. A total of $>50 \%$ positively stained tumor cells were scored as $3,30-50 \%$ as $2,10-30 \%$ as 1 and $<10 \%$ as 0 . The final scores were evaluated by multiplying the intensity score by the percentage score: 0 , negative $(-) ; 1-2$, weakly positive $(1+) ; 3-5$, moderately positive $(2+)$; and 6-9, strongly positive $(3+)$.
Statistical analysis. The positive expression levels of ANGPTL4 among normal breast, ADH, DCIS and IDC tissues were analyzed using $\chi^{2}$ test with Bonferroni correction. The distribution of ANGPTL4 expression in patients with breast cancer according to clinicopathological features was analyzed using the non-parametric Kruskal-Wallis test. Multiple comparisons among the groups were performed using the Nemenyi test. The Kaplan-Meier method was used to evaluate the association between ANGPTL4 expression and 
Table III. Cox proportional hazards regression analysis for DFS and OS.

\begin{tabular}{|c|c|c|c|c|c|c|}
\hline \multirow[b]{2}{*}{ Parameters } & \multicolumn{3}{|c|}{ DFS } & \multicolumn{3}{|c|}{ OS } \\
\hline & P-value & Relative risk & $95 \% \mathrm{CI}$ & P-value & Relative risk & $95 \% \mathrm{CI}$ \\
\hline Age, years $(<55$ vs. $\geq 55)$ & 0.066 & 1.644 & $0.240-3.217$ & 0.075 & 1.234 & $0.256-2.768$ \\
\hline ER (negative vs. positive) & 0.110 & 0.440 & $0.161-1.205$ & 0.210 & 0.330 & $0.330-1.789$ \\
\hline PR (negative vs. positive) & 0.059 & 0.324 & $0.101-1.042$ & 0.078 & 0.225 & $0.134-1.567$ \\
\hline HER2 ( 0 and $1+$ vs. $2+$ and $3+)$ & 0.943 & 1.014 & $0.733-1.403$ & 0.956 & 1.002 & $0.567-1.876$ \\
\hline ANGPTL4 (negative vs. positive) & 0.011 & 2.353 & $0.733-3.403$ & 0.034 & 2.034 & $0.534-3.567$ \\
\hline pTNM stage (I+II vs. III+IV) & 0.004 & 2.450 & $1.338-4.488$ & 0.002 & 2.678 & $1.448-4.213$ \\
\hline Grade (I+II vs. III) & 0.524 & 0.764 & $0.378-2.468$ & 0.645 & 0.534 & $1.456-2.145$ \\
\hline Tumor size, $\mathrm{cm}(\leq 5$ vs. $>5)$ & 0.876 & 1.075 & $0.435-2.656$ & 0.896 & 0.989 & $0.546-2.132$ \\
\hline Lymph node metastasis (no vs. yes) & 0.021 & 1.844 & $1.098-3.099$ & 0.043 & 1.234 & $0.987-3.007$ \\
\hline
\end{tabular}

DFS, disease-free survival; OS, overall survival; ER, estrogen receptor; PR, progesterone receptor; HER2, human epidermal growth factor receptor 2; ANGPTL4, angiopoietin-like 4.

A

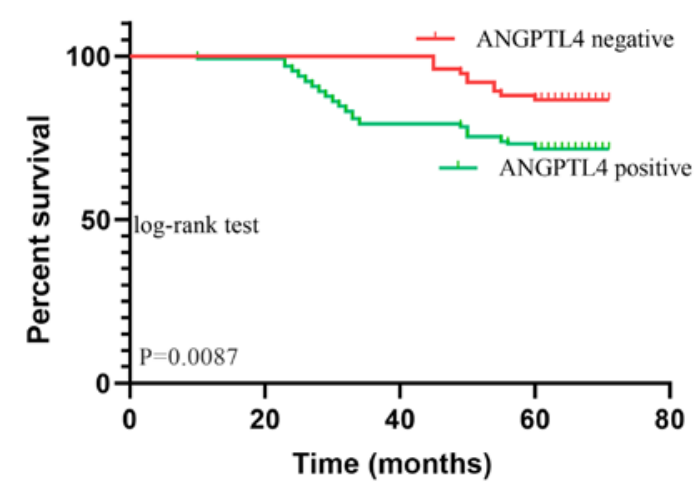

B

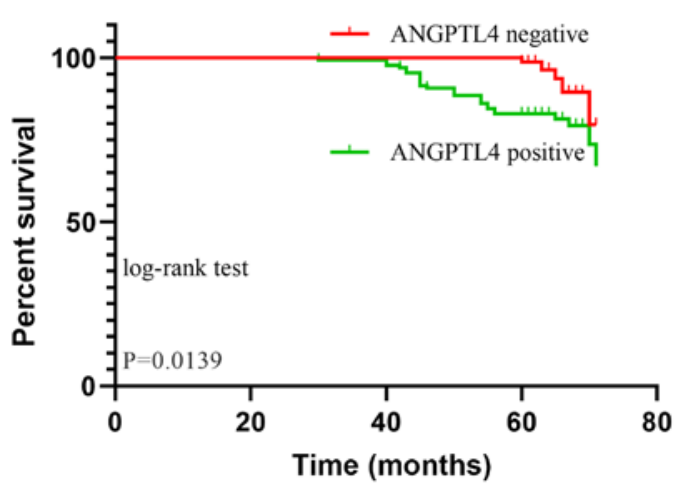

Figure 3. Survival curves based on ANGPTL4 expression. (A) DFS and (B) OS analysis of ANGPTL4 in breast cancer. ANGPTL4, angiopoietin-like 4; DFS, disease-free survival; OS, overall survival.

breast cancer prognosis, and differences in survival distributions were evaluated using the log-rank test. Cox regression analysis was used to identify the factors that may have a significant influence on survival. Statistical analyses were performed using SPSS v17.0 (SPSS, Inc.). P<0.05 was considered to indicate a statistically significant difference.

\section{Results}

Differential expression of ANGPTL4 in normal breast tissues, $A D H, D C I S$ and IDC. The expression levels of ANGPTL4 were analyzed in 40 normal breast, 40 ADH, 40 DCIS and 205 IDC tissues. Notably, ANGPTL4 was expressed differentially in normal, ADH, DCIS and IDC tissues. The positive expression rates of ANGPTL4 were $17.5 \%$ (7/40), 30.0\% (12/40), 37.5\% $(15 / 40)$ and $63.0 \%(130 / 205)$ in normal, ADH, DCIS and IDC tissues, respectively (Table I). The expression patterns in the different tissues are shown in Figs. 1 and 2.

Association between ANGPTL4 expression and clinical features of IDC. The clinical significance of ANGPTL4 expression was analyzed in 205 IDC tissues. High expression levels of ANGPTL4 in breast cancer were positively associated with pathological stage, tumor size, grade, lymph node metastasis, distant metastasis and local recurrence (Table II). No association was identified between ANGPTL4 expression and age, and estrogen receptor, progesterone receptor and human epidermal growth factor receptor 2 expression (Table II).

Association between ANGPTL4 expression and prognosis of breast cancer. In the analyzed cohort, there were 12 patients with recurrence, 37 patients with metastasis and six patients with both recurrence and metastasis. A total of 28 patients died of breast cancer and three patients died of other reasons. Kaplan-Meier analysis of the present cohort of patients with breast cancer demonstrated significant differences in DFS between patients with breast cancer with negative expression of ANGPTL4 and those with positive expression of ANGPTL4 $(\mathrm{P}=0.0087)$. Additionally, the OS between patients with negative ANGPTL4 expression and those with positive ANGPTL4 expression was significantly different ( $\mathrm{P}=0.0139$; Fig. 3). ANGPTL4 expression was associated with poor prognosis in patients with breast cancer.

Variables with $\mathrm{P}<0.05$ in the univariate Cox regression analysis were included in the multivariate analysis. Multivariate Cox regression analysis revealed that pathological stage, lymph node metastasis and ANGPTL4 expression 
were significant prognostic factors for breast cancer. Patients with positive ANGPTL4 expression had 2.353 and 2.034-fold higher relative risks of DFS and OS, respectively, than those with negative ANGPTL4 expression (Table III).

\section{Discussion}

Previous studies have demonstrated that ANGPTL4 is involved in cancer growth and progression, angiogenesis, metabolism and metastasis $(11,22,23)$. In a number of cancer types, the diverse roles of ANGPTL4 remain controversial and are mainly attributed to different cellular system targets, such as the microenvironment of the tumor and endothelial cells. One theory is that ANGPTL4 expression is increased by hypoxia-inducible factor 1- $\alpha$ (HIF-1 $\alpha)$-stimulated tumorigenesis, which has been demonstrated in Kaposi's sarcoma (24). Additionally, a previous study demonstrated that ANGPTL4 upregulation in endothelial cells expressing $\mathrm{G}$ protein-coupled receptors serves a key role in the tumorigenesis of Kaposi's sarcoma, supporting the aforementioned results (25). Another theory is that ANGPTL4 acts via the transforming growth factor $\beta$ (TGF- $\beta$ ) signaling pathway to promote tumor metastasis (26). Controversially, another study has reported that ANGPTL4 serves an anti-angiogenic role as a suppressor of tumorigenesis (24). The pro- or anti-angiogenic effects of ANGPTL4 are associated with the tumor microenvironment. The role of ANGPTL4 in human cancer is most likely complex. In addition to angiogenesis, ANGPTL4 is involved in inflammation and energy metabolism depending on the context of the cancer (9). In colorectal cancer, pre-clinical and clinical studies have demonstrated that ANGPTL4 overexpression promotes cell migration and invasion $(9,15)$. Additionally, ANGPTL4 has been revealed to promote metastasis in oral cancer (27). These findings suggest that ANGPTL4 may be a potential biomarker and therapeutic target for oral squamous cell carcinoma and colorectal cancer. Additionally, ANGPTL4 may serve a diagnostic and prognostic role for patients with hepatic cell cancer and renal cell carcinoma, and may be used as a potential biomarker for future targeted therapies $(28,29)$. In preclinical models, treatment with anti-ANGPTL4 antibody blocks angiogenesis, motility and metastasis by destroying the tumor-favorable microenvironment (28). In vitro, the downregulation of ANGPTL4 and simultaneous upregulation of fibronectin 1 have been implicated in the antitumor activity of the oncosuppressor U94 in androgen-resistant prostate cancer cell lines (30). Additionally, ANGPTL4 is associated with the AKT signaling pathway, which is critical in breast cancer, and clinical trials of AKT small molecule inhibitors are ongoing. The role that ANGPTL4 serves in breast cancer has attracted considerable attention. Wei et al (31) reported that inhibiting ANGPTL4 in a breast cancer cell line could inhibit cancer cell invasion. However, to the best of our knowledge, the mechanism by which ANGPTL4 promotes metastasis in breast cancer has not been established. One study reported that TGF- $\beta$ primes breast tumors for lung metastasis seeding via ANGPTL4 (32). Another study reported that ANGPTL4 promotes breast cancer metastasis via the HIF-1 signaling pathway (33). Although this remains controversial, this phenomenon was consistent with the results of the present study.

The present study demonstrated that ANGPTL4 served a key role in breast cancer. First, IHC staining was used to analyze 40 normal breast, 40 ADH, 40 DCIS and 205 breast cancer tissues, and the results demonstrated that as the degree of malignancy in epithelial breast tissue increased, the positive rate of ANGPTL4 expression also increased gradually. This suggested that ANGPTL4 can be regarded as a gene closely-associated with breast cancer.

To validate the role of ANGPTL4 in breast cancer, the expression levels of this protein were analyzed by IHC in 205 samples of patients with breast cancer. The results of the present study revealed that ANGPTL4 served an important role in the malignant progression of breast cancer. Furthermore, ANGPTL4 expression was positively associated with poor prognostic factors. In vivo and in vitro models in previous studies of other tumors have demonstrated the ability of ANGPTL4 to regulate cell-cell and cell-matrix communication, cell migration, invasion and metastatic dissemination $(34,35)$, which is consistent with the findings of the present study in breast cancer.

Finally, the association between ANGPTL4 expression and prognosis in 205 patients with breast cancer was analyzed. The current results revealed that positive expression of ANGPTL4 was associated with poor DFS and OS. Cox regression analysis demonstrated that ANGPTL4 is an independent prognostic factor for breast cancer, suggesting that ANGPTL4 may be a novel therapeutic target for breast cancer.

Although the function of ANGPTL4 has been controversial in different types of cancer, the present study was, to the best of our knowledge, the first to analyze ANGPTL4 expression in breast cancer tissues and to demonstrate that it was associated with the malignant progression and poor prognosis of breast cancer. Previous studies have reported inconsistent mechanisms mediated by ANGPTL4 in breast cancer $(36,37)$. The present study suggested a potential carcinogenic and pre-metastatic role for ANGPTL4, which needs to be further elucidated in future studies.

\section{Acknowledgements}

Not applicable.

\section{Funding}

No funding was received.

\section{Availability of data and materials}

The datasets used and/or analyzed during the current study are available from the corresponding author on reasonable request.

\section{Authors' contributions}

MW and JZ designed the study. JZ and $\mathrm{HZ}$ collected the clinical data. JZ analyzed and interpreted the data. JL, SZ, NW and LL acquired and prepared the clinical samples. JL supervised the study. JZ and HZ wrote, reviewed and/or revised the manuscript. All authors read and approved the final manuscript.

\section{Ethics approval and consent to participate}

The present study was approved by the Ethics Committee of the Cancer Institute \& Hospital of Tianjin Medical University 
(Tianjin, China; approval no. EK2012053). Written informed consent was provided by all patients at the time of tissue collection.

\section{Patient consent for publication}

Not applicable.

\section{Competing interests}

The authors declare that they have no competing interests.

\section{References}

1. Tao Z, Shi A, Lu C, Song T, Zhang Z and Zhao J: Breast cancer: Epidemiology and etiology. Cell Biochem Biophys 72: 333-338, 2015.

2. Hoon Tan P, Ellis I, Allison K, Brogi E, Fox SB, Lakhani S, Lazar AJ, Morris EA, Sahin A, Salgado R, et al: The 2019 WHO classification of tumours of the breast. Histopathology: Feb 13 2020 (Epub ahead of print).

3. Emens LA: Breast cancer immunotherapy: Facts and hopes. Clin Cancer Res 24: 511-520, 2018.

4. Ge W, Jiang M, Zhang F, Ma Y, Wang $\mathrm{H}$ and Xu Y: ZGRF1 is associated with poor prognosis in triple-negative breast cancer and promotes cancer stemness based on bioinformatics. Onco Targets Ther 13: 2843-2854, 2020.

5. Aljohani AI, Joseph C, Kurozumi S, Mohammed OJ, Miligy IM, Green AR and Rakha EA: Myxovirus resistance 1 (MX1) is an independent predictor of poor outcome in invasive breast cancer. Breast Cancer Res Treat 181: 541-551, 2020.

6. Zhou Z, Yao H and $\mathrm{Hu} \mathrm{H}$ : Disrupting tumor angiogenesis and 'the hunger games' for breast cancer. Adv Exp Med Biol 1026 : 171-195, 2017.

7. Oike Y, Yasunaga K, Ito Y, Matsumoto S, Maekawa H, Morisada T, Arai F, Nakagata N, Takeya M, Masuho Y and Suda T: Angiopoietin-related growth factor (AGF) promotes epidermal proliferation, remodeling, and regeneration. Proc Natl Acad Sci USA 100: 9494-9499, 2003.

8. Oike Y, Ito Y, Maekawa H, Morisada T, Kubota Y, Akao M, Urano T, Yasunaga $\mathrm{K}$ and Suda T: Angiopoietin-related growth factor (AGF) promotes angiogenesis. Blood 103: 3760-3765, 2004

9. Carbone C, Piro G, Merz V, Simionato F, Santoro R, Zecchetto C, Tortora G and Melisi D: Angiopoietin-like proteins in angiogenesis, inflammation and cancer. Int J Mol Sci 19: 431, 2018.

10. McQueen AE, Kanamaluru D, Yan K, Gray NE, Wu L, Li ML, Chang A, Hasan A, Stifler D, Koliwad SK and Wang JC: The C-terminal fibrinogen-like domain of angiopoietin-like 4 stimulates adipose tissue lipolysis and promotes energy expenditure. J Biol Chem 292: 16122-16134, 2017.

11. Tan MJ, Teo Z, Sng MK, Zhu P and Tan NS: Emerging roles of angiopoietin-like 4 in human cancer. Mol Cancer Res 10: 677-688, 2012

12. Chen JW, Luo YJ, Yang ZF, Wen LQ and Huang L: Knockdown of angiopoietin like 4 inhibits the development of human gastric cancer. Oncol Rep 39: 1739-1746, 2018.

13. Tanaka J, Irie T, Yamamoto G, Yasuhara R, Isobe T, Hokazono C, Tachikawa T, Kohno Y and Mishima K: ANGPTL4 regulates the metastatic potential of oral squamous cell carcinoma. J Oral Pathol Med 44: 126-133, 2015.

14. Zhang $\mathrm{H}$, Wei S, Ning S, Jie Y, Ru Y and Gu Y: Evaluation of TGF $\beta$, XPO4, elF5A2 and ANGPTL4 as biomarkers in HCC. Exp Ther Med 5: 119-127, 2013.

15. Nakayama T, Hirakawa H, Shibata K, Nazneen A, Abe K, Nagayasu T and Taguchi T: Expression of angiopoietin-like 4 (ANGPTL4) in human colorectal cancer: ANGPTL4 promotes venous invasion and distant metastasis. Oncol Rep 25: 929-935, 2011.

16. Dong D, Jia L, Zhou Y, Ren L, Li J and Zhang J: Serum level of ANGPTL4 as a potential biomarker in renal cell carcinoma. Urol Oncol 35: 279-285, 2017.

17. Wang Y, Liu LM, Wei L, Ye WW, Meng XY, Chen F, Xiao Q, Chen JY and Zhou Y: Angiopoietin-like protein 4 improves glucose tolerance and insulin resistance but induces liver steatosis in high-fat-diet mice. Mol Med Rep 14: 3293-3300, 2016.
18. Nitulescu GM, Van De Venter M, Nitulescu G, Ungurianu A, Juzenas P, Peng Q, Olaru OT, Grădinaru D, Tsatsakis A, Tsoukalas D, et al: The Akt pathway in oncology therapy and beyond (Review). Int J Oncol 53: 2319-2331, 2018.

19. Nitulescu GM, Margina D, Juzenas P, Peng Q, Olaru OT, Saloustros E, Fenga C, Spandidos DA, Libra M and Tsatsakis AM: Akt inhibitors in cancer treatment: The long journey from drug discovery to clinical use (Review). Int J Oncol 48: 869-885, 2016.

20. Mundi PS, Sachdev J, McCourt C and Kalinsky K: AKT in cancer: New molecular insights and advances in drug development. Br J Clin Pharmacol 82: 943-956, 2016.

21. Amin MB, Edge SB, Greene FL, et al (eds): AJCC cancer staging manual. 8th edition. Springer, New York, NY, 2017.

22. Cazes A, Galaup A, Chomel C, Bignon M, Bréchot N, Le Jan S, Weber H, Corvol P, Muller L, Germain S and Monnot C: Extracellular matrix-bound angiopoietin-like 4 inhibits endothelial cell adhesion, migration, and sprouting and alters actin cytoskeleton. Circ Res 99: 1207-1215, 2006.

23. Galaup A, Cazes A, Le Jan S, Philippe J, Connault E, Le Coz E, Mekid H, Mir LM, Opolon P, Corvol P, et al: Angiopoietin-like 4 prevents metastasis through inhibition of vascular permeability and tumor cell motility and invasiveness. Proc Natl Acad Sci USA 103: 18721-18726, 2006.

24. La Paglia L, Listì A, Caruso S, Amodeo V, Passiglia F, Bazan V and Fanale D: Potential role of ANGPTL4 in the cross talk between metabolism and cancer through PPAR signaling pathway. PPAR Res 2017: 8187235, 2017.

25. Hu J, Jham BC, Ma T, Friedman ER, Ferreira L, Wright JM, Accurso B, Allen CM, Basile JR and Montaner S: Angiopoietin-like 4: A novel molecular hallmark in oral Kaposi's sarcoma. Oral Oncol 47: 371-375, 2011.

26. Mathieu M, Iampietro M, Chuchana P, Guérit D, Djouad F, Noël D and Jorgensen C: Involvement of angiopoietin-like 4 in matrix remodeling during chondrogenic differentiation of mesenchymal stem cells. J Biol Chem 289: 8402-8412, 2014.

27. Huang Z, Xie J, Lin S, Li S, Huan Z, Wang Y and Ye J: The downregulation of ANGPTL4 inhibits the migration and proliferation of tongue squamous cell carcinoma. Arch Oral Biol 71: 144-149, 2016.

28. Ng KT, Xu A, Cheng Q, Guo DY, Lim ZX, Sun CK, Fung JH, Poon RT, Fan ST, Lo CM and Man K: Clinical relevance and therapeutic potential of angiopoietin-like protein 4 in hepatocellular carcinoma. Mol Cancer 13: 196, 2014.

29. Verine J, Lehmann-Che J, Soliman H, Feugeas JP, Vidal JS, Mongiat-Artus P, Belhadj S, Philippe J, Lesage M, Wittmer E, et al: Determination of Angptl4 mRNA as a diagnostic marker of primary and metastatic clear cell renal-cell carcinoma. PLoS One 5: e10421, 2010.

30. Ifon ET, Pang AL, Johnson W, Cashman K, Zimmerman S, Muralidhar S, Chan WY, Casey J and Rosenthal LJ: U94 alters FN1 and ANGPTL4 gene expression and inhibits tumorigenesis of prostate cancer cell line PC3. Cancer Cell Int 5: 19, 2005.

31. Wei Z, Shan Y, Tao L, Liu Y, Zhu Z, Liu Z, Wu Y, Chen W, Wang $A$ and Lu Y: Diallyl trisulfides, a natural histone deacetylase inhibitor, attenuate HIF-1 $\alpha$ synthesis, and decreases breast cancer metastasis. Mol Carcinog 56: 2317-2331, 2017.

32. Padua D, Zhang XH, Wang Q, Nadal C, Gerald WL, Gomis RR and Massagué J: TGFbeta primes breast tumors for lung metastasis seeding through angiopoietin-like 4. Cell 133: 66-77, 2008.

33. Zhang H, Wong CC, WeiH, Gilkes DM,Korangath P, Chaturvedi $\mathrm{P}$, Schito L, Chen J, Krishnamachary B, Winnard PT Jr, et al: HIF-1-dependent expression of angiopoietin-like 4 and L1CAM mediates vascular metastasis of hypoxic breast cancer cells to the lungs. Oncogene 31: 1757-1770, 2012.

34. Liao YH, Chiang KH, Shieh JM, Huang CR, Shen CJ, Huang WC and Chen BK: Epidermal growth factor-induced ANGPTL4 enhances anoikis resistance and tumour metastasis in head and neck squamous cell carcinoma. Oncogene 36: 2228-2242, 2017.

35. Sun Y, Long J and Zhou Y: Angiopoietin-like 4 promotes melanoma cell invasion and survival through aldolase A. Oncol Lett 8: 211-217, 2014.

36. Gong X, Hou Z, Endsley MP, Gronseth EI, Rarick KR, Jorns JM, Yang Q, Du Z, Yan K, Bordas ML, et al: Interaction of tumor cells and astrocytes promotes breast cancer brain metastases through TGF-32/ANGPTL4 axes. NPJ Precis Oncol 3: 24, 2019.

37. Tripathy J, Tripathy A, Thangaraju M, Suar M and Elangovan S: $\alpha$-Lipoic acid inhibits the migration and invasion of breast cancer cells through inhibition of TGF $\beta$ signaling. Life Sci 207: 15-22, 2018. 